\section{FRI0688 PREDICTORS OF EROSION AND JOINT SPACE NARROWING PROGRESSION IN PATIENTS WITH EARLY RHEUMATOID ARTHRITIS}

Emil Rydell ${ }^{1,2}$, Kristina Forslind ${ }^{3,4}$, Jan-Åke Nilsson ${ }^{1,2}$, Lennart T.H. Jacobsson ${ }^{1,5}$, Carl Turesson ${ }^{1,2} .{ }^{1}$ Rheumatology, Department of Clinical Sciences, Malmö, Lund University, Malmö, Sweden; ${ }^{2}$ Department of Rheumatology, Skåne University Hospital, Malmö, Sweden; ${ }^{3}$ Rheumatology, Department of Clinical Sciences, Helsingborg, Lund University, Helsingborg, Sweden; ${ }^{4}$ Research and Education, Helsingborg Hospital, Helsingborg, Sweden; ${ }^{5}$ Rheumatology and Inflammation Research, Sahlgrenska Academy at Gothenburg University, Göteborg, Sweden

Background: Joint damage in rheumatoid arthritis (RA) includes erosions and joint space narrowing (JSN). Predictors of these processes, and the underlying mechanisms, require further study ${ }^{1}$.

Objectives: To investigate the relation between patient characteristics at RA diagnosis and progression of erosions and JSN, over 5 years.

Methods: Consecutive early RA patients (symptom duration $<12$ months), recruited 1995-2005 from a defined area, managed according to usual care with no pre-specified treatment protocol, were followed through 5 years. Radiographs of hands and feet were scored in chronological order by a trained reader according to the modified Sharp-van der Heijde score (SHS), including separate erosion- and JSN scores. The relations between baseline variables and progression of erosion- and JSN scores over 5 years (log transformed), were assessed using linear regression.

Results: 233 early RA patients where included. Radiographs at baseline and 5 years were available for 162 patients. Results on predictors of rapid radiographic progression of the total SHS have been reported previously $^{2}$. The median (interquartile) progression of erosion and JSN scores were $4(0-8)$ and 8 (1-16), respectively. RF and anti-CPP predicted progression of erosions and JSN over 5 years, with stronger associations for erosions (Table 1). Baseline erosion- and JSN scores each predicted progression of JSN, while baseline JSN did not predict progression of erosions. In crude analyses, higher disease activity, CRP and ESR were associated with progression of both erosions and JSN, while statistically more robust for the former. Smoking and high levels of Cartilage Oligomeric Matrix Protein (COMP) (>12 U/L) were both associated with progression of erosions $((B=0.12, p=0.016)$ and $(B=0.12, p=0.02)$ respectively) but not JSN, in adjusted analyses (Table 1). Overweight or obesity $\left(\mathrm{BMI}>25 \mathrm{~kg} / \mathrm{m}^{2}\right)$ was associated with less progression of $\mathrm{JSN}(\mathrm{B}=$ $-0.14, p=0.018$, adjusted for RF, age and baseline JSN score).

Table 1. Baseline predictors of progression of erosion and JSN scores (log transformed) from baseline to 5 years in linear regression

\begin{tabular}{|c|c|c|c|c|c|c|c|c|}
\hline \multirow{3}{*}{ Baseline characteristics } & \multicolumn{4}{|c|}{ Erosion score } & \multicolumn{4}{|c|}{ JSN score } \\
\hline & \multicolumn{2}{|c|}{ Crude } & \multicolumn{2}{|c|}{$\begin{array}{l}\text { Adjusted for } \\
\text { RF and } \\
\text { baseline } \\
\text { erosion } \\
\text { score }\end{array}$} & \multicolumn{2}{|c|}{ Crude } & \multicolumn{2}{|c|}{$\begin{array}{c}\text { Adjusted for } \\
\text { RF, age and } \\
\text { baseline } \\
\text { JSN score }\end{array}$} \\
\hline & $\mathrm{B}$ & $p$ & B & $p$ & $B$ & $p$ & $B$ & $\mathrm{p}$ \\
\hline Age (per SD) & 0.02 & 0.39 & 0.02 & 0.45 & 0.06 & 0.051 & NA & NA \\
\hline $\begin{array}{l}\text { Overweight or obese vs. } \\
\text { normal BMI }\end{array}$ & -0.05 & 0.34 & -0.03 & 0.62 & -0.14 & 0.03 & -0.14 & 0.018 \\
\hline Ever vs. never smokers & 0.16 & 0.004 & 0.12 & 0.016 & 0.05 & 0.49 & 0.06 & 0.33 \\
\hline RF positivity & 0.23 & $<0.001$ & NA & NA & 0.18 & 0.004 & NA & NA \\
\hline Anti-CCP positivity & 0.23 & $<0.001$ & 0.13 & 0.02 & 0.17 & 0.008 & 0.09 & 0.18 \\
\hline COMP > $12 \mathrm{U} / \mathrm{L}$ & 0.10 & 0.08 & 0.12 & 0.02 & 0.08 & 0.24 & 0.08 & 0.23 \\
\hline Erosion score (per SD) & 0.07 & 0.006 & NA & NA & 0.12 & $<0.001$ & 0.08 & 0.02 \\
\hline JSN score (per SD) & 0.04 & 0.12 & -0.01 & 0.69 & 0.11 & $<0.001$ & NA & NA \\
\hline $\begin{array}{l}\text { High vs. moderate } \\
\text { DAS2 }{ }^{\text {b }}\end{array}$ & 0.17 & 0.003 & 0.12 & 0.03 & 0.14 & 0.04 & 0.12 & 0.054 \\
\hline ESR (per SD) & 0.11 & $<0.001$ & 0.08 & 0.002 & 0.09 & 0.003 & 0.06 & 0.04 \\
\hline CRP $>9 \mathrm{mg} / \mathrm{l}(\text { median })^{\mathrm{C}}$ & 0.15 & 0.003 & 0.09 & 0.08 & 0.13 & 0.03 & 0.07 & 0.26 \\
\hline
\end{tabular}

Conclusion: RF, anti-CCP and markers of inflammation and disease activity predicted progression of erosions and JSN, in particular erosions. Development of erosions may predate cartilage damage leading to JSN. Smoking and high baseline levels of COMP predicted progression of erosions, but not JSN. Overweight and obesity may be associated with mechanisms that protect from JSN.

\section{REFERENCES:}

[1] Smolen et al, Ann Rheum Dis. 2009;68:1535-40.2 Rydell et al, Arthritis Res Ther. 2018;20:82.
Disclosure of Interests: Emil Rydell: None declared, Kristina Forslind: None declared, Jan-Åke Nilsson: None declared, Lennart T.H. Jacobsson Consultant for: LJ has received lecture and consulting fees from Pfizer, Abbvie, Novartis, Eli-Lily and Janssen, Carl Turesson: None declared DOI: 10.1136/annrheumdis-2019-eular.381

\section{FRI0689 EFFECT OF AIR POLLUTION EXPOSURE ON DISEASE ACTIVITY IN RHEUMATOID ARTHRITIS}

Tommaso Schioppo ${ }^{1}$, Isabella Scotti ${ }^{1}$, Simona lodice ${ }^{2}$, Mirjam Hoxha ${ }^{2}$, Orazio De Lucia ${ }^{1}$, Antonella Murgo ${ }^{1}$, Valentina Bollati ${ }^{2}$, Francesca Ingegnoli ${ }^{1,2}{ }^{1}{ }^{1} A S S T$ Pini-CTO, Division of Clinical Rheumatology, Milano, Italy; ${ }^{2}$ University of Milan, Department of Clinical Sciences and Community Health, Milano, Italy

Background: Environmental exposure (e.g. tobacco smoking, pollution) has been accepted to contribute in autoimmune diseases. Air pollution has been described to be a risk factor for developing rheumatoid arthritis (RA). Currently, RA disease remission is considered an achievable target in a significant proportion of patients. Nevertheless, diseases flares, that significantly contributes to damage progression and disability, remain unpredictable. Thus, factors able to potentially interfere on disease activity should be considered and assessed.

Objectives: The aim of our study is to evaluate the influence of particulate matter (PM) on disease activity and general health $(\mathrm{GH})$ in patients with RA.

Methods: Consecutive patients with RA (ACR/EULAR Criteria 2010) resident in Lombardy (Italy) were enrolled. In each patient Disease Activity Score on 28 joints (DAS28), Simple Disease Activity Index (SDAI) and patients' GH were assessed. Daily PM concentrations, estimated by Regional Environmental Protection Agency at municipality resolution, were used to assign short-term exposure from day of visit back to 14 days Continuous variables are expressed as mean $\pm S D$. Categorical variables are presented as absolute numbers and frequencies. Multivariable linear regression models were performed to identify the day of PM10 and PM2.5 independently associated with DAS28, GH and SDAI indices, adjusting for the variables significant at the univariate analysis. $\mathrm{GH}$ was log transformed to achieve normality of residuals. $\beta$ coefficients were reported for $10 \mu \mathrm{g} / \mathrm{m} 3$ increments of $P M$ concentrations. All statistical analyses were performed using SAS 9.4

Results: 258 patients (age at visit $57.4 \pm 13.5$ years, disease duration 16.2 \pm 12.1 , female $80.2 \%$, rheumatoid factor and/or anti-citrullinated peptide antibodies positivity $60.5 \%$, smokers $15.5 \%$, radiographic damage $41.5 \%$ ) were enrolled in the study. Multivariable linear regression models were adjusted for radiographic damage, disease duration and age. Increases of PM2.5 and PM10 exposure (9 day before the visit) were significantly associated with worsening of DAS28, SDAI and GH (table below). PM concentrations in days other than 9 days before were significantly associated with disease activity and $\mathrm{GH}$.

\begin{tabular}{|l|c|c|c|c|c|c|}
\hline & PM Exposure & $\boldsymbol{\beta} \times 10$ & SE & \multicolumn{2}{|c|}{ 95\% CI } & P-value \\
\hline \multirow{2}{*}{ DAS28 } & $\mathrm{PM}_{10}$ Day -9 & 0,10 & 0,05 & 0,00 & 0,19 & 0,041 \\
\cline { 2 - 7 } & $\mathrm{PM}_{2.5}$ Day -9 & 0,11 & 0,06 & 0,00 & 0,23 & 0,048 \\
\hline \multirow{2}{*}{ SDAI } & $\mathrm{PM}_{10}$ Day -9 & 0,80 & 0,39 & 0,02 & 1,57 & 0,043 \\
\cline { 2 - 7 } & $\mathrm{PM}_{2.5}$ Day -9 & 0,95 & 0,48 & 0,00 & 1,90 & 0,049 \\
\hline \multirow{2}{*}{ GH } & $\mathrm{PM}_{10}$ Day -9 & 0,11 & 0,04 & 0,03 & 0,18 & 0,004 \\
\cline { 2 - 7 } & $\mathrm{PM}_{2.5}$ Day -9 & 0,14 & 0,05 & 0,06 & 0,23 & 0,002 \\
\hline
\end{tabular}

Conclusion: In our cohort, PM10 and PM2.5 exposure seems to influence RA disease activity with a lag period that can last until 9 days before the visit. Nevertheless, the association between day-to-day PM changes and disease activity do not confirm causation. Further studied are required to evaluate the influence of air pollution on RA activity. Disclosure of Interests: None declared

DOI: 10.1136/annrheumdis-2019-eular.4822 